\title{
Thermal and hydrostatic structure of the protoplanetary disks: Influences of wind strengths, mass distributions, and stellar wind velocity laws
}

\author{
Young Seok Yun ${ }^{1}$, Hiroyuki Emori ${ }^{2}$, and Kiyoshi Nakazawa ${ }^{3}$ \\ ${ }^{1}$ VARAVON Inc.811 Mecha zone, 2979 Hagun-ri Yangchon-myeon, Gimpo-si, Gyeonggi-do, Korea \\ ${ }^{2}$ Department of Education, Shumei University, Chiba 276-0003, Japan \\ ${ }^{3}$ Department of Earth and Planetary Sciences, School of Science, Tokyo Institute of Technology, Tokyo 152-8551, Japan
}

(Received September 28, 2010; Revised July 17, 2012; Accepted December 18, 2012; Online published August 23, 2013)

\begin{abstract}
The structures of the protoplanetary disk have been examined under various conditions of the stellar wind and the mass distribution of the disk, by assuming that the disk is steady and geometrically thick. T Tauri stars are commonly accompanied by disks as well as the stellar wind. Therefore, the disk around a T Tauri star should be influenced by the stellar wind. The height of the geometrical surface of the disk is suppressed by the dynamical pressure of the wind but depends very weakly on the wind strength. The surface becomes slightly higher when the wind strength becomes weaker. Furthermore, the dependency on the mass distribution of the disk is also weak. As a natural result, the temperature distribution in the disk is insensitive to the wind strength and also the mass distribution of the disk. Thus, we can conclude that the temperature and the geometrical surface height of the disk under the stellar wind does not depend on either the wind properties or the mass distribution of the disk. Key words: Passive disk, mass loss rate, stellar wind, mass distribution.
\end{abstract}

\section{Introduction}

It is commonly believed that planets form in a disk that passively receives and reflects radiation from a central star. Therefore, in the study of planetary formation, it is necessary to obtain a good grasp of the physical properties which provide information about the initial conditions of subsequent planetary formation processes. In particular, it is most important to know the temperature distribution over the entire disk, because this governs the distribution of the solid mass in the disk at the beginning of planetary formation.

During the last two or three decades, astronomical observations have revealed a sequence of stellar formation processes, from protostars to pre-main-sequence stars (e.g., Shu et al., 1987). Among young stellar objects, such as the protostars and $\mathrm{T}$ Tauri stars, we are particularly interested in T Tauri stars, because their ages almost correspond to the characteristic planetary formation time (e.g., Hayashi et al., 1985). The development of observational studies on disks surrounding young stellar objects has stimulated theoreticians who have investigated various aspects of protoplanetary disks: emission features from young stellar objects at various stages (e.g., Beckwith et al., 1990; Hartmann and Kenyon, 1990; Calvet et al., 1992; Edwards et al., 1994; Chiang et al., 2001); the dynamical and thermal structure of a protoplanetary disk (Kusaka et al., 1970, which is referred to as K70 hereafter, and Chiang and Goldreich, 1997, which is referred to as CG97 hereafter); the gas temperature and the chemical structure of a protoplanetary disk

Copyright (C) The Society of Geomagnetism and Earth, Planetary and Space Sciences (SGEPSS); The Seismological Society of Japan; The Volcanological Society of Japan; The Geodetic Society of Japan; The Japanese Society for Planetary Sciences; TERRAPUB.

doi:10.5047/eps.2012.12.009 (e.g., Kamp and Dullemond, 2004; Jonkheid et al., 2004); the thermal instability due to flaring of a passive disk (e.g., D'Alessio et al., 1999; Watanabe and Lin, 2008) and due to self-shadowing (e.g., Dullemond, 2000; Dullemond et al., 2001; Dullemond and Domink, 2004); and the mass distribution in the disk (Hayashi, 1981).

Among these subjects, we put a priority on studies of the temperature structure in the passive disk in which the first physical (and also chemical) processes of planetary formation would begin. The temperature in the disk essentially determines the mass and the chemical composition of solid materials in the disk. If the disk temperature is higher than $170 \mathrm{~K}$ (which is the freezing temperature of ice under the typical gas pressure; e.g., Hayashi et al., 1985), only rocky and metallic materials can condense to form solid particles. On the other hand, if the temperature is below the freezing point of ice, solid particles containing a great amount of ice can exist. The mass of solid particles in the region where solid ice exists would be about four times larger than that in the region where ice sublimes (Hayashi et al., 1985). It is very important to specify the place where ice condenses, namely, the position of the snow line in a protoplanetary disk, because the snow line governs the boundary between regions of the terrestrial-type planets and the Jovian-type planets (e.g., Sasselov and Lecar, 2000; Garaud and Lin, 2007). Furthermore, it should also be pointed out that the temperature distribution of a protoplanetary disk governs the thermal and dynamical instability of the disk (e.g., D'Alessio et al., 1999; Dullemond and Domink, 2004; Watanabe and Lin, 2008).

Despite its importance, however, there are few theoretical works, to date, on the temperature distribution of the passive disk. K70 is a pioneering work on the temperature distribu- 
tion of the disk determined by solar radiation onto its surface. Another temperature model of the passive disk around T Tauri stars was presented by CG97. They took into account an optically thin, slightly high-temperature region on the top of the disk and evaluated the radiation reprocessed by this surface layer to heat the interior disk. Contrary to these highly-elaborate calculations applied to consider the spectral feature of T Tauri stars, there is, unfortunately, an omission in the works mentioned above. Namely, they constructed their thermal models for passive protoplanetary disks without regard to the fact that $\mathrm{T}$ Tauri stars would be accompanied by a stellar wind.

Kuhi (1964) recognized the presence of winds from $\mathrm{T}$ Tauri stars by analyzing in detail their emission profiles. Nowadays, it is widely accepted that most of the classical $\mathrm{T}$ Tauri stars have an extremely strong wind (e.g., Carr, 1989; Strom et al., 1989; Greene and Mayer, 1995; McCaughrean and O'Dell, 1996). From extensive observations it has become clear that the strong wind from a T Tauri star is closely related to disk accretion because of the strong correlation between the mass loss rates and the mass accretion rates (Cabrit et al., 1990; Calvet et al., 1992; Hartigan et al., 1995). As a protoplanetary disk evolves from an active phase (from classical T Tauri stars) to a passive phase (to weak-line T Tauri stars), the stellar wind from the central star may change from a strong accretion-driven wind to a weak wind (e.g., Kenyon and Hartmann, 1995; Calvet, 1997; Wood et al., 2002). On the mass loss rates of the stellar wind in the passive phase, some observational evidence shows that these are under $1 \times 10^{-7} M_{\odot} \mathrm{yr}^{-1}$ (Hartmann et al., 1990; Hartigan et al., 1995; Calvet, 1997), or as weak as $1 \times 10^{-8} M_{\odot} \mathrm{yr}^{-1}$ (Cabrit et al., 1990; Hartigan et al., 1995; White and Hillenbrand, 2004). It can readily be imagined that if the disk around the central star has a hydrostatic state the stellar wind from the star might blow the stellar wind with mass loss rates nearly equal to, or weaker than, $1 \times 10^{-8} M_{\odot} \mathrm{yr}^{-1}$.

Yun et al. (2007; which is called Y07 hereafter) reconstructed a model of the passive disk exposed to both the stellar radiation and the stellar wind. Although Y07 is the first work in which the effect of the stellar wind is taken into account, their study has been found wanting for the following reasons;

(1) They limited themselves to the case where the surface density of the disk depends on the radial distance from the central star $r$ as $r^{-\frac{3}{2}}$.

(2) They limited themselves to the case where the wind velocity is constant.

(3) The work has a problem concerning the basic equations (Yun et al., 2010).

As will be seen in the next section, the basic equations contain an important parameter that characterize the radial distribution of the disk mass. So, as a natural extension of Y07, we will make clear the effect of this parameter on the disk temperature. The aim of the present study is to investigate the thermal behavior of the protoplanetary disk exposed to stellar radiation and the stellar wind over a wide range of three principal parameters: the strength of the stellar wind, the parameters describing the mass distribution of the disk, and the velocity law of the stellar wind.

In Section 2, we will describe the assumptions adopted as well as the basic equations. The contents of Section 2 are nearly the same as those of Y70 (note that a basic equation is revised following Yun et al., 2010).

In Section 3, we describe the thermal and the dynamical structure of a disk. First, we will see in detail the effect of the wind strength under the fixed parameters describing the disk mass distribution and the velocity distribution of the stellar wind. Next, we will comment briefly on the similarity of the disk models, taking into account the difference in mass distribution of the disk and of the wind velocity variation. From the descriptions in Section 3, we will see that, surprisingly, the disk models are very similar to each other irrespective of the wide variety in the considered parameters. Finally, conclusions and remarks are presented in Section 4.

\section{Assumptions and Basic Equations}

We consider a protoplanetary disk in the passive phase, exposed to the wind and radiation from a central $\mathrm{T}$ Tauri star. As a preparation for obtaining the geometrical figure and the temperature distribution of the disk, we will describe briefly the assumptions made and the basic equations, since the contents are very similar to those of Y07. In this study, the mass distribution of the disk and the velocity law of the stellar wind are somewhat extended compared with those of Y07, and the dynamical balance equation is revised because the equation used in Y07 is incorrect (see Yun et al., 2010).

\subsection{Adopted assumptions}

As Y07, we assume that the disk is axis-symmetric and mirror-symmetric with respect to the mid-plane of the disk. In addition to that, the disk is in a hydrostatic equilibrium state and is isothermal in the $z$-direction (see figure 1 of Y07). Furthermore, the disk is assumed to be geometrically very thin and is optically thick. Formally, the radial mass distribution of the disk is given by the same law as in the case of Y07, i.e., by a power-law function of $r$ with an index $\gamma$ :

$$
\Sigma=\Sigma_{0}\left(\frac{r}{1 \mathrm{AU}}\right)^{\gamma},
$$

where $\Sigma$ is the surface density of the disk and $\Sigma_{0}$ is that at $r=1 \mathrm{AU}$. In Y07, $\gamma$ is fixed to be $-3 / 2$ but, in this study, $\gamma$ is treated as a parameter. Furthermore, we adopt a simple model for the stellar wind, as in Y07, supposing that the wind is steady and spherically symmetric.

\subsection{Basic equations}

As seen in Y07, there are three basic equations: an auxiliary equation describing the disk height and two balance equations. Let $z_{\mathrm{s}}$ be the height of the disk, i.e., the height of the contact surface between the disk and the stellar wind. The disk height, $z_{\mathrm{s}}$, is described by:

$$
\frac{d z_{\mathrm{s}}}{d r}=\frac{r f+z_{\mathrm{s}} \sqrt{1-f^{2}}}{r \sqrt{1-f^{2}}-z_{\mathrm{s}} f},
$$

where $f$ is the flaring index (see Y07).

Two physical equations are derived from the dynamical and thermal balances on the disk surface. Here, we assume 
that on the contact surface between the disk and the stellar wind, the normal component of the dynamical pressure balances the hydrostatic pressure in the disk. Thus, we have:

$$
P_{\mathrm{w} \perp}=P,
$$

where $P_{\mathrm{w} \perp}$ is the normal component of the dynamical pressure, $P_{\mathrm{w}}$, of the stellar wind at the contact surface. Under the assumptions mentioned in the previous subsection, the hydrostatic pressure of the disk at height $z$ can be written as:

$$
P=\frac{k T}{\mu m_{\mathrm{H}}} \frac{\Sigma}{\sqrt{2 \pi} H} \exp \left(-\frac{z^{2}}{2 H^{2}}\right),
$$

where $T$ is the disk temperature, $k$ is the Boltzmann constant, and $m_{\mathrm{H}}$ is the mass of a hydrogen atom. In the above, we assume that the disk is composed of an ideal gas with a mean molecular weight, $\mu=2.34$, and $\sqrt{2} H$ is the pressure scale height of the disk given by:

$$
\sqrt{2} H=r\left(\frac{2 k T r}{\mu m_{\mathrm{H}} G M_{*}}\right)^{\frac{1}{2}}
$$

where $M_{*}$ is the mass of the central star.

Furthermore, the dynamical pressure of the wind, $P_{\mathrm{w}}$, is given by

$$
P_{\mathrm{w}}=\frac{M_{*}}{4 \pi r^{2}} \frac{v_{\mathrm{w}}}{t_{*}}
$$

In the above, $v_{\mathrm{w}}$ is the wind velocity which can be expressed by means of the escape velocity from the central star as:

$$
v_{\mathrm{w}}=\beta(r)\left(\frac{2 G M_{*}}{R_{*}}\right)^{\frac{1}{2}},
$$

where $R_{*}$ is the radius of the central star and $\beta(r)$ is a certain dimensionless function of $r$. Furthermore, $t_{*}$ is the typical mass loss time of the central star, defined as:

$$
t_{*} \equiv \frac{M_{*}}{\dot{M}_{\mathrm{w}}}
$$

where $\dot{M}_{\mathrm{w}}$ is the mass loss rate due to the wind from the central star.

Using the flaring index, $f$, of the contact surface, Eq. (3) can be rewritten as:

$$
P=f^{2} P_{\mathrm{w}}
$$

at $z=z_{\mathrm{s}}$, where $P$ and $P_{\mathrm{w}}$ are given by Eqs. (4) and (6), respectively (see figure 1 in Y07). In the above, we have to explain the reason why the degree of the flaring index $f$ is equal to 2. Firstly, the momentum flux of the wind received by a contact surface of unit area is reduced by a factor of $f$ because the cross-section normal to the momentum direction is equal to $f$. Secondly, the component of the momentum flux normal to the contact surface is given by a further decrease of a factor of $f$ (see Yun et al., 2010).

Assuming that the contact surface is approximately a blackbody surface at which the disk absorbs the stellar luminosity and radiates the thermal emission, we use the following equation for the energy balance between the stellar irradiation from a central star and the blackbody radiation from the surface (e.g., K70):

$$
\begin{aligned}
\sigma T^{4}= & \frac{\sigma T_{*}^{4}}{\pi} \frac{R_{*}^{2}}{r^{2}}\left\{\frac{\pi}{2} f \frac{1+z_{\mathrm{s}} / r}{\sqrt{1+\left(z_{\mathrm{s}} / r\right)^{2}}}\right. \\
& +\frac{2 R_{*}}{3 r}\left[8 f \frac{z_{\mathrm{s}} / r}{1+\left(z_{\mathrm{s}} / r\right)^{2}}\right. \\
& \left.\left.+\frac{1-d z_{\mathrm{s}}^{2} / d r^{2}}{\sqrt{1+\left(d z_{\mathrm{s}} / d r\right)^{2}} \sqrt{1+\left(z_{\mathrm{s}} / r\right)^{2}}}\right]\right\},
\end{aligned}
$$

where $\sigma$ is the Stefan-Boltzmann constant, and $T_{*}$ is the photospheric temperature of the central star. On the right hand side of this equation, the first term in the outer parentheses represents the heating due to the flaring of the disk, and the second term represents the contribution due to the fact that the central star has a finite radius. In the Appendix, we present the derivation of Eq. (10) in detail.

\subsection{Non-dimensional forms of the basic equations}

The structure of the disk exposed to the stellar radiation, as well as the stellar wind, is completely determined by three equations: the dynamical and thermal balances described by Eqs. (9) and (10), respectively, and the supplementary geometrical condition given by Eq. (2). To avoid complexity, let us introduce dimensionless variables and rewrite the basic equations. We replace the radial distance, $r$, the disk height, $z_{\mathrm{s}}$, and the disk temperature, $T$, by dimensionless variables $\eta, v$, and $u$, respectively:

$$
\eta=\frac{r}{R_{*}}, \quad v=\frac{z_{\mathrm{s}}}{r}, \quad \text { and } \quad u=\frac{T}{T_{*}}\left(2 \eta^{2}\right)^{\frac{1}{4}} .
$$

Furthermore, we introduce two dimensionless parameters which are given by:

$N=2^{\frac{1}{4}} \frac{T_{\mathrm{g}}}{T_{*}}$ and $M=2^{\frac{7}{8}} \sqrt{\pi} \mathrm{G} \Sigma_{0} \sqrt{\frac{R_{*}}{2 \mathrm{G} M_{*}}} t_{*}\left(\frac{T_{*}}{T_{\mathrm{g}}}\right)^{\frac{1}{2}}\left(\frac{R_{*}}{1 \mathrm{AU}}\right)^{\gamma}$,

where $T_{\mathrm{g}}$ is the temperature corresponding to the gravitational potential energy at the stellar surface; originally introduced by CG97 as:

$$
T_{\mathrm{g}}=\frac{\mathrm{G} M_{*} \mu m_{\mathrm{H}}}{2 k R_{*}} .
$$

Both the flaring index, $f$, and the dimensionless disk height, $v$, are very small $\left(f \ll 1\right.$ and $z_{\mathrm{s}} / r \ll 1$ ) because of the thin disk approximation. Hence, we can neglect terms of $\left(z_{\mathrm{s}} / r\right)^{2},\left(d z_{\mathrm{s}} / d r\right)^{2}, d z_{\mathrm{s}}^{2} / d r^{2}, f^{2}$, and $f\left(z_{\mathrm{s}} / r\right)$, compared to unity in the basic equations. Thus, using these variables and parameters, we have:

$$
\begin{gathered}
\frac{d v}{d \eta}=\frac{f}{\eta}, \\
f^{2}=M \eta^{\gamma+\frac{1}{4}} \frac{1}{\beta(\eta)} \sqrt{u} \exp (-s),
\end{gathered}
$$

and

$$
u^{4}=f+\frac{4}{3 \pi} \frac{1}{\eta},
$$


where

$$
s \equiv N \frac{v^{2}}{\sqrt{\eta} u} .
$$

Combining Eqs. (14) to (17), we obtain an equation for the flaring index, $f$, which is given by:

$$
\mathcal{L}(f, \eta) \frac{d \ln f}{d \ln \eta}=\mathcal{R}(f, \eta)
$$

where

$$
\mathcal{L}(f, \eta)=2-\frac{f}{8 u^{4}}(1+2 s)
$$

and

$\mathcal{R}(f, \eta)=\gamma+\frac{1}{4}-\frac{d \ln \beta}{d \ln \eta}-\frac{1}{8 u^{4}} \frac{4}{3 \pi \eta}(1+2 s)+\frac{s}{2}\left(1-4 \frac{f}{v}\right)$.

As mentioned above, one of the basic equations of our previous study Y07 is revised here. Nevertheless, the topological behavior of the solutions to Eq. (18) is very similar to that in Y07 as long as we are concerned with an inner disk of less than $50 \mathrm{AU}$. Thus, we can obtain a suitable disk structure by the same method as in Y07.

\section{Results}

In this section, we present the results which are obtained by solving numerically the set of basic equations described in Section 2 over a wide range of wind strength, as well as the radial mass distribution parameter $\gamma$ (see Eq. (1)). We also mention briefly the results for the case of the wind model with a radially-dependent wind velocity. For the central star, we choose $T_{*}=4000 \mathrm{~K}, R_{*}=2 R_{\odot}$, and $M_{*}=1 M_{\odot}$. As for the inner and the outer boundaries, we take these to be $0.03 \mathrm{AU}(\eta=3)$ and $50 \mathrm{AU}\left(\eta=5 \times 10^{3}\right)$.

\subsection{Disk models with different wind strengths}

We will first present disk models for various strengths of the stellar wind. Throughout the present subsection, we adopt the Hayashi model (Hayashi, 1981) in which $\Sigma_{0}=1.7 \times 10^{3} \mathrm{~g} / \mathrm{cm}^{2}$ and $\gamma=-3 / 2$ for the radial mass distribution of the disk and constant wind velocity. Accordingly, we set $\beta(r)=\beta$ in Eq. (7) and change $\beta$ as a parameter. As for the wind strength, we consider the following five cases:

$$
\begin{aligned}
t_{*} / \beta= & 1 \times 10^{8}, 1 \times 10^{9}, 1 \times 10^{10}, 1 \times 10^{11}, \\
& \text { and } 1 \times 10^{12} \mathrm{yr} .
\end{aligned}
$$

Though the last four cases were studied in Y07, we recalculated these cases using the revised Eq. (15).

In Fig. 1, we illustrate the flaring index, $f$, as a function of $\eta$ for the five cases of the wind parameters $t_{*} / \beta$ between $1 \times 10^{8} \mathrm{yr}$ and $1 \times 10^{12} \mathrm{yr}$. As seen in Y07, the profile of the flaring index $f$ changes for each case of the adopted value of $t_{*} / \beta$. Although, we can say that it is very small in all cases of the wind parameter at any distance from the central star. Furthermore, it becomes small with an increase in wind strength because the stronger wind suppresses the surface of the disk more strongly by the dynamic pressure of the wind.

The temperature distributions of the disk are plotted in Fig. 2(a) for the wind parameters from $1 \times 10^{8}$ yr to $1 \times$

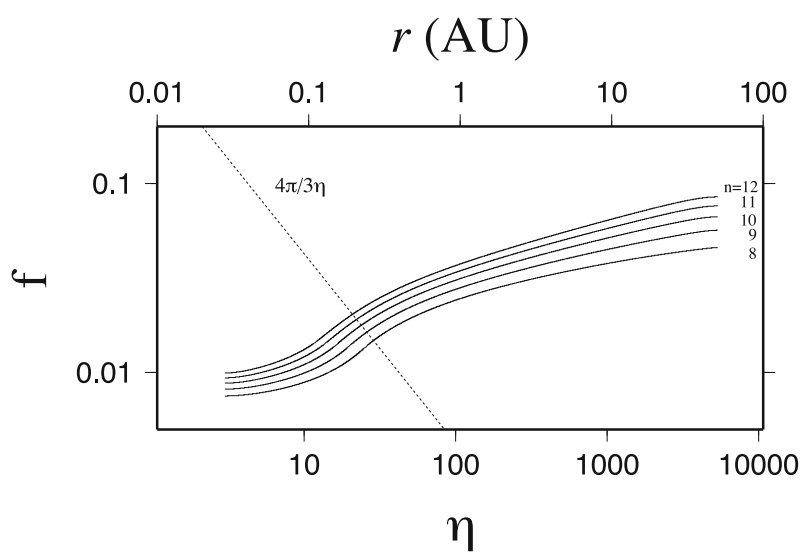

Fig. 1. The flaring index, $f$, as a function of $\eta$ for five different cases of $t_{*} / \beta$. In the figure, $n$ denotes the result for the case of $t_{*} / \beta=1 \times 10^{n} \mathrm{yr}$. We also overwrite the second term, $4 / 3 \pi \eta$, in the right hand side of Eq. (16) in order to readily see the roles in determining the disk temperature.

Table 1. The disk temperatures and their logarithmic derivatives (i.e., $-d \ln T / d \ln r)$ at $r=0.1 \mathrm{AU}$ and $1 \mathrm{AU}$ for the wind parameters from $1 \times 10^{8}$ yr to $1 \times 10^{12} \mathrm{yr}$.

\begin{tabular}{l||c|c|c|c}
\hline \multicolumn{1}{c||}{} & \multicolumn{2}{c|}{$T_{0}[\mathrm{~K}]$} & \multicolumn{2}{c}{$p_{0}$} \\
\cline { 2 - 5 }$t_{*} / \beta[\mathrm{yr}]$ & $0.1[\mathrm{AU}]$ & $1[\mathrm{AU}]$ & $0.1[\mathrm{AU}]$ & $1[\mathrm{AU}]$ \\
\hline $1 \times 10^{8}$ & 481 & 133 & 0.690 & 0.478 \\
\hline $1 \times 10^{9}$ & 484 & 137 & 0.682 & 0.473 \\
\hline $1 \times 10^{10}$ & 487 & 141 & 0.673 & 0.468 \\
\hline $1 \times 10^{11}$ & 490 & 144 & 0.663 & 0.465 \\
\hline $1 \times 10^{12}$ & 493 & 146 & 0.653 & 0.462 \\
\hline
\end{tabular}

$10^{12} \mathrm{yr}$. From this figure, we can see that the temperature distribution depends very weakly on the wind parameter $t_{*} / \beta$. Especially, in the inner region of the disk $(r \leq$ $0.1 \mathrm{AU})$, the temperature distributions of the disk for five different wind parameters coincide almost perfectly with each other, because the temperature is determined mainly by radiative heating due to the finite size of the central star in this region. Inspecting in detail, we can identify the suppressed temperature throughout the disk for the strong wind as same as the smallness of the flaring index $f$ (see Eq. (16) and also Fig. 1). Details of the temperatures are tabulated numerically in Table 1 at typical distances from the central star, $r=0.1 \mathrm{AU}$ and $1 \mathrm{AU}$, for the adopted wind parameters $t_{*} / \beta$. The temperatures at these distances can be approximately expressed as functions of the wind parameter; i.e.,

$T=481+3.0 \log \left\{\left(t_{*} / \beta\right) / 1 \times 10^{8} \mathrm{yr}\right\} \mathrm{K} \quad$ at $\quad r=0.1 \mathrm{AU}$,

and

$T=133+3.5 \log \left\{\left(t_{*} / \beta\right) / 1 \times 10^{8} \mathrm{yr}\right\} \mathrm{K} \quad$ at $\quad r=1 \mathrm{AU}$.

These equations show quantitatively how weakly the disk temperature depends on the wind parameter.

The logarithmic derivatives of the temperature, $p$, defined by $-d \ln T / d \ln r$, are shown in Fig. 2(b) for the wind parameters, and the values of $p$ at the distances $0.1 \mathrm{AU}$ and 1 AU are tabulated in Table 1. From the results under the 

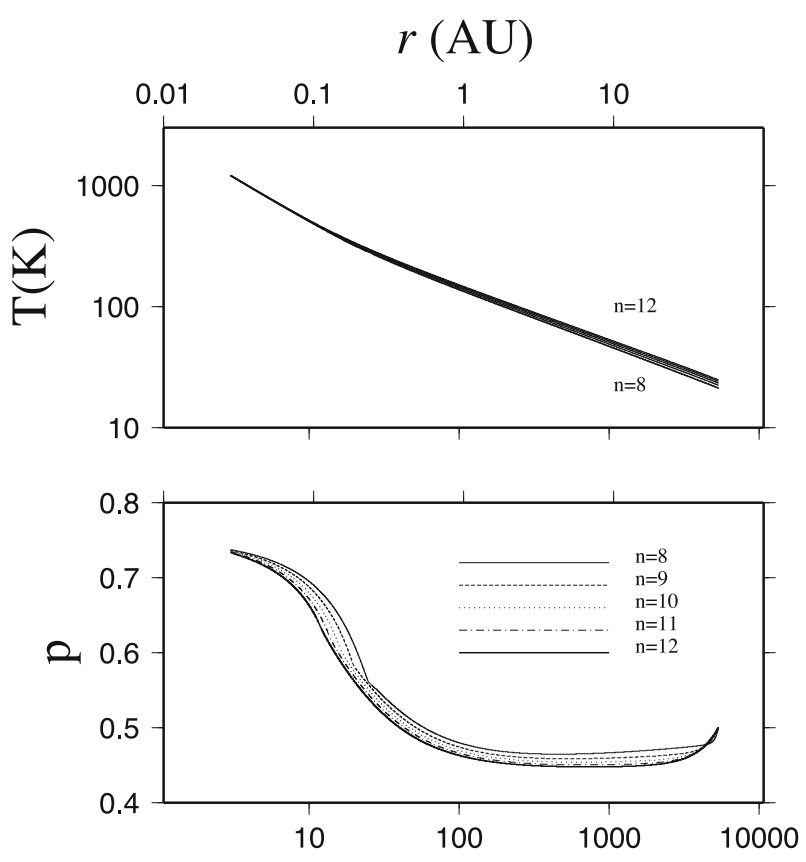

$\eta$

Fig. 2. Temperature distributions of the disk for the five cases of $t_{*} / \beta$ from $1 \times 10^{8}$ to $1 \times 10^{12} \mathrm{yr}$ (panel (a)). We also show the corresponding power-law index $p$ defined as $p=-d \ln T / d \ln r$ in panel (b). In the figure, the index $n$ represents the case of $t_{*} / \beta=1 \times 10^{n} \mathrm{yr}$.

five conditions of the wind, we can confirm that the index $p$ becomes larger for the stronger wind case in most regions of the disk.

In the intermediate region between $0.1 \mathrm{AU}$ and $1 \mathrm{AU}$, $p$ changes abruptly but, in the inner $(r \leq 0.1 \mathrm{AU})$ and the outer $(r \geq 1 \mathrm{AU})$ regions, the variation of $p$ is small. Therefore, the temperature distribution may be approximately described by a power-law function as long as we are concerned with the inner or outer regions of the disk; that is,

$T(r) \propto T_{0}\left(\frac{r}{r_{0}}\right)^{p_{0}} \mathrm{~K} \quad$ for $\quad r \leq 0.1 \mathrm{AU} \quad$ or $\quad r \geq 1 \mathrm{AU}$,

where $T_{0}$ and $p_{0}$ are the temperature and the logarithmic derivative tabulated in Table 1. In the case that we consider the temperature in the inner region, we set $r_{0}$ equal to 0.1 $\mathrm{AU}$, and in the case for the outer region we use $1 \mathrm{AU}$. As a result, roughly speaking, the temperature distributions in the inner and the outer regions can be approximated by power-law functions given by

$$
T(r)= \begin{cases}480(r / 0.1 \mathrm{AU})^{-3 / 4} \mathrm{~K} & \text { for } \quad r \leq 0.1 \mathrm{AU} \\ 135(r / 1 \mathrm{AU})^{-1 / 2} \mathrm{~K} & \text { for } \quad r \geq 1 \mathrm{AU}\end{cases}
$$

These are quite similar to equations (24) and (25) in Y07.

The heights of the disk surface are shown in Fig. 3(a) for five cases of $t_{*} / \beta$ from $1 \times 10^{8} \mathrm{yr}$ to $1 \times 10^{12} \mathrm{yr}$. At a glance, we see that the disk heights do not depend so much on the parameter $t_{*} / \beta$. All lines are confined within a factor of 1.5 except the narrow region near the outer boundary. Furthermore, we also see that $\log z_{\mathrm{s}}$ increases with $\log \eta$ almost linearly. This enables us to conjecture that the heights are well described as a power-law function of the radial posi-
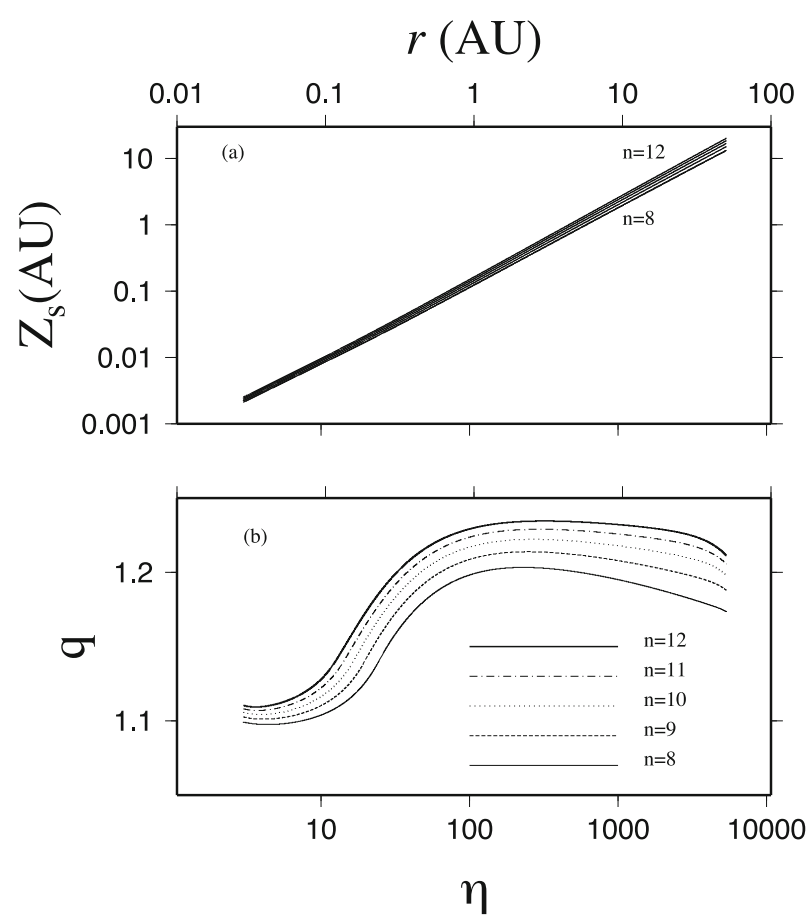

Fig. 3. The disk heights, $z_{\mathrm{s}}$, for the five cases of $t_{*} / \beta$ (panel(a)) and the power-law index, $q$, i.e., $d \ln z_{\mathrm{s}} / d \ln r(\operatorname{panel}(\mathrm{b}))$. In the figure, the symbol $n$ has the same meaning as in Fig. 2.

tion $r$. In order to see the dependence more clearly, we plot the power index $q$ defined as $q=d \ln z_{\mathrm{s}} / d \ln r$ in Fig. 3(b). Similarly as with the temperature distribution, the height of the disk surface depends on the stellar wind strength a little. As the wind parameter $t_{*} / \beta$ becomes larger, the height of the disk surface becomes greater.

As an example of the weak or moderate wind cases, let us consider the line corresponding to $t_{*} / \beta=1 \times 10^{10}$. From Fig. 3(b), we see that the power-law index $q$ of the disk height is almost constant, namely, about 1.1 in the inner region. As the distance from the central star becomes larger than $0.1 \mathrm{AU}$, the index $q$ begins to increase and reaches about 1.2 at $r=1 \mathrm{AU}$. After that the index ceases to increase and becomes almost constant again. We obtain similar results even in other cases of $t_{*} / \beta$. On the basis of these results, we can find approximate power-law functions describing $z_{\mathrm{s}} / r$. Namely, we have

$$
\frac{z_{\mathrm{s}}}{r}= \begin{cases}0.10(r / 0.1 \mathrm{AU})^{0.1} & \text { for } \quad r \leq 0.1 \mathrm{AU} \\ 0.15(r / 1 \mathrm{AU})^{0.2} & \text { for } \quad r \geq 1 \mathrm{AU}\end{cases}
$$

with an accuracy of $10 \%$ (for the case of $r=0.1 \mathrm{AU}$, the precise value of $z / r$ is 0.09 for $t_{*} / \beta=1 \times 10^{8} \mathrm{yr}$ and 0.11 for $1 \times 10^{12} \mathrm{yr}$ ).

Finally, in Fig. 4, we present spectral energy distributions from the temperature distribution for three cases of mass loss rate. We assume that the disk is perpendicular to the sight line from us to the star and we employ the same procedure as in Miyake and Nakagawa (1995) to represent the SED that would be observed. We cannot recognize a clear difference between the three cases where $t_{*} / \beta$ is $1 \times 10^{12}, 1 \times 10^{10}$, and $1 \times 10^{8}$ yr. So it is difficult to identify the mass loss rates from spectral information. 


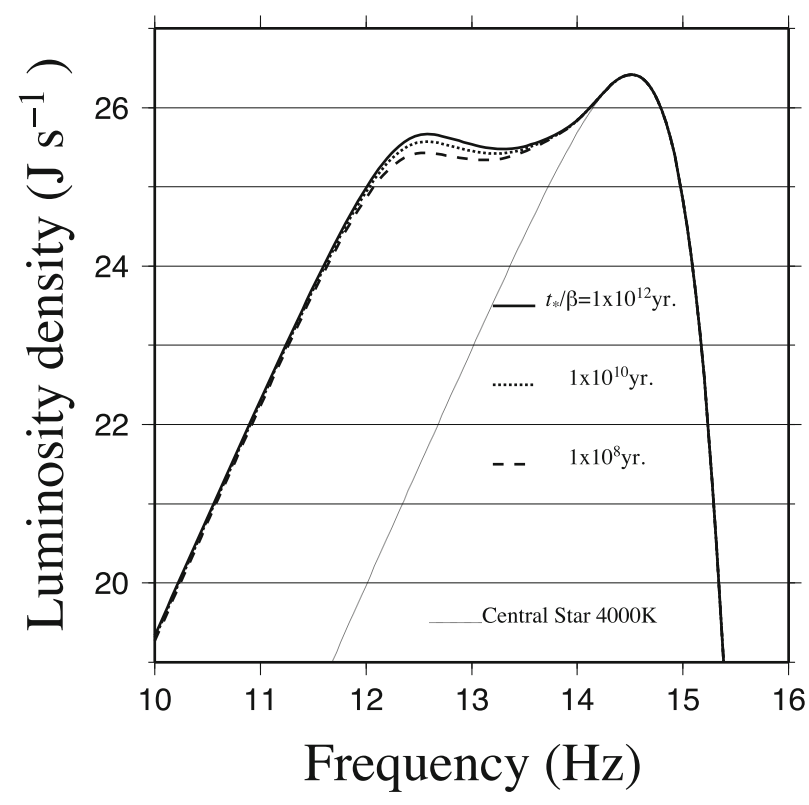

Fig. 4. Spectral energy distributions for 3 protoplanetary disks which have the surface temperature presented in Fig. 2(a). Mach mass loss times are equal to $1 \times 10^{12}, 1 \times 10^{10}$, and $1 \times 10^{8} \mathrm{yr}$.

\subsection{Different mass distributions in the disk}

In this subsection, we will briefly consider how the adopted disk mass distribution affects the thermal and dynamical structure of the disk. As in the previous subsection, we assume that the surface density of the disk is given by a power law of $r$, i.e., the form of Eq. (1). As for $\gamma$, we consider the following four cases:

$$
\gamma=-\frac{5}{4}, \quad-\frac{4}{3}, \quad-\frac{3}{2}, \quad \text { and }-2,
$$

but $\Sigma_{0}$ is fixed to the previous value, $1.7 \times 10^{3} \mathrm{~g} / \mathrm{cm}^{2}$. It should be noted here that the surface mass density at $1 \mathrm{AU}$ is a constant independent of the adopted value of $\gamma$ and that the surface density in the inner region increases with a decrease in $\gamma$ and, on the other hand, decreases in the region outside $1 \mathrm{AU}$. In the present subsection, the wind parameter, $t_{*} / \beta$, is commonly put to be $1 \times 10^{12} \mathrm{yr}$.

The results are shown in Fig. 5. Roughly speaking, the flaring index, $f$, the disk height, $z_{\mathrm{s}}$, and the disk temperature, $T$, are all identical regardless of the adopted value of $\gamma$. In order to see in detail the obtained feature shown in Fig. 5, and to grasp as a whole the effect of $\gamma$ on the structure of disk, it may be better to consider the panels of Fig. 5 in the inverse order. In the inner region, where $r \leq 0.1 \mathrm{AU}$, the temperature is determined uniquely by the second term of Eq. (16), i.e., $4 / 3 \pi \eta$, and, hence, is never influenced by $\gamma$. On the other hand, in the outer region the temperature is governed mainly by $f^{1 / 4}$ (see Eq. (16)) and, if $f$ does not depend so much on $\gamma$, the temperature becomes almost independent of $\gamma$ (as will be seen later, this is so in our present case) as seen Fig. 5(c).

The height of the disk surface, $z_{\mathrm{s}}$, is shown in Fig. 5(b) for four cases of $\gamma . z_{\mathrm{s}}$ increases with an increase in $\gamma$ in the inner region, whereas it decreases with $\gamma$ in the outer region. But we can safely say that the height behaves similarly for all the case of $\gamma$ taken here. Note that the height

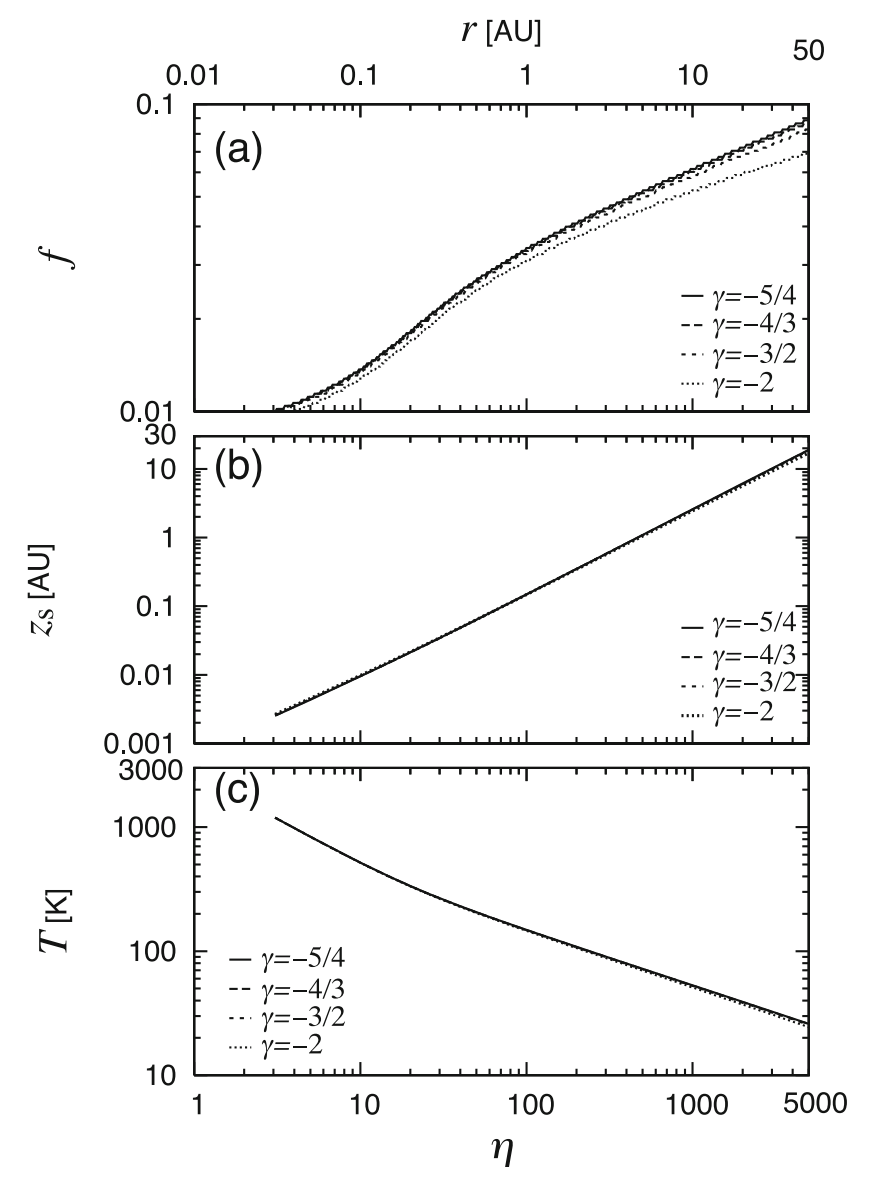

Fig. 5. Dependence of the flaring index, $f$ (panel (a)), the disk height, $z_{\mathrm{s}}$ (panel (b)), and the temperature, $T$ (panel (c)), on the power-law index $\gamma$ which characterizes the mass distribution (see Eq. (1)). As for $t_{*} / \beta$, we take this as $1 \times 10^{12} \mathrm{yr}$.

of the disk surface is determined by a balance between the dynamical pressure of the wind and the gas pressure in the disk which is proportional to the local matter density. As mentioned earlier, the surface density in the outer region ( $r \geq 1$ AU) behaves in an inverse way to that in the inner region ( $r \leq 1 \mathrm{AU}$ ) when the power index $\gamma$ changes. As a result, in the inner region the gas pressure increases and, hence, the height $z_{\mathrm{s}}$ becomes higher when $\gamma$ is larger. In the outer region the surface density and the gas pressure decrease. So the height of the disk becomes lower with an increment in the power index $\gamma$.

As seen from Fig. 5(a), the flaring index, $f$, does not change in the inner region ( $r \leq 1 \mathrm{AU}$ ) but in the outer region, where $r \geq 1 \mathrm{AU}$, there is a notable discrepancy in $f$ for the case of $\gamma=-2$. As mentioned in the previous section, $f$ is approximately proportional to $z_{\mathrm{s}} / r$ in the outer region where the first term of the right hand side of Eq. (16) becomes important (see Fig. 1). Thus, we can say that the decline in $f$ is caused by that in $z_{\mathrm{s}}$.

Anyway, the dependency on $\gamma$ of the flaring index $f$, and the height $z_{\mathrm{s}}$ of the disk, is very weak. Furthermore, the temperature does not depend on the radial mass distribution of the disk. This is also true for the other cases of $t_{*} / \beta$, as long as we are concerned with cases where $t_{*} / \beta$ is greater than $1 \times 10^{8} \mathrm{yr}$. 


\subsection{Influence of the adopted stellar wind model}

In the previous two subsections, we assumed that, for simplicity, the stellar wind velocity is constant over the whole region between the inner and the outer boundaries of the disk. Unfortunately, the velocity distribution of the stellar wind is not detected observationally so that it is natural to consider that the wind velocity varies as a function of the distance from a central star. Y07 tried to calculate the temperature and the height of the disk, as an example, using the velocity model proposed by Parker $(1960,1964)$. First, we will recalculate the structure of the disk under the same conditions as $\mathrm{Y} 07$, since an incorrect basic equation was used. As with the calculation presented in Y07, we consider the case only of $t_{*}=1 \times 10^{12} \mathrm{yr}$ (which corresponds to $\dot{M}_{\mathrm{w}}=1 \times 10^{-12} M_{\odot} \mathrm{yr}^{-1}$; see Eq. (8)). In this case, the wind velocity, $v_{\mathrm{p}}$, of Parker's solution is shown in Fig. 6(a). The wind velocity $v_{\mathrm{p}}$ is expressed as a ratio to $v_{\mathrm{c}}$ that is introduced as the sound velocity corresponding to the coronae temperature. For the case of $\beta(r)=1$, the velocity, $v_{\mathrm{c}}$, is $4.37 \times 10^{2} \mathrm{~km} / \mathrm{s}$. In other words, $v_{\mathrm{c}}$ is almost equal to the escape velocity from the central star (see Eq. (7)). We compare models with the same parameters of the wind and the central star. As seen from this figure, there is not so much difference between $v_{\mathrm{p}}$ and $v_{\mathrm{c}}$. Namely, $v_{\mathrm{p}} / v_{\mathrm{c}}$ is about $1 / 3$ at the innermost region ( $r \sim 0.03 \mathrm{AU})$ and about 2 at the outermost region $(r \sim 50 \mathrm{AU})$. Furthermore, we adopt the power-law model of the mass distribution with the index $\gamma$ equal to $-3 / 2$.

In Figs. 6(b) and 6(c), we illustrate the temperature and the height of the disk, respectively, which are calculated numerically by the use of Parker's solution. For comparison, we also give the results based on the constant velocity wind model with $v_{\mathrm{c}}$ as shown by dashed curves. Obviously the two models give almost the same results (as presented by Y07), though the height of the disk is very slightly suppressed in the outer region $(r \geq 30 \mathrm{AU})$, where $\left(v_{\mathrm{p}} / v_{\mathrm{c}}\right)^{2}$ is larger than 2. Anyway, we can say that the results described in Subsection 3.1 are not changed even under the stellar wind expressed by Parker's solution, since we can also confirm that we have obtained similar results for the other cases of the mass loss rate.

To make doubly sure of our conclusions, we consider another wind model in which the wind velocity decreases with radial distance from a central star, as opposed to that of Parker's model. As a typical example, we adopt a velocity law of particles (with zero energy) which are influenced only by the gravity of the central star, that is, $\beta(r)$ in Eq. (7) is put to be:

$$
\beta(r)=\left(\frac{R_{*}}{r}\right)^{1 / 2} .
$$

Note that, in the present wind model, the wind pressure $P_{\mathrm{w}}$ is reduced by a factor of $\beta(r)$ compared with the case of the original constant velocity wind model with $\beta(r)=1$. If the same mass loss rate is imposed for both cases (see Eqs. (6) and (8)) $\beta(r)$ is as small as about $1 / 10$ and $1 / 70$ at a distance of $1 \mathrm{AU}$ and $50 \mathrm{AU}$ from the central star, respectively.

Using Eq. (28), we calculate the distributions of the temperature and the height of the disk. The results are illus-
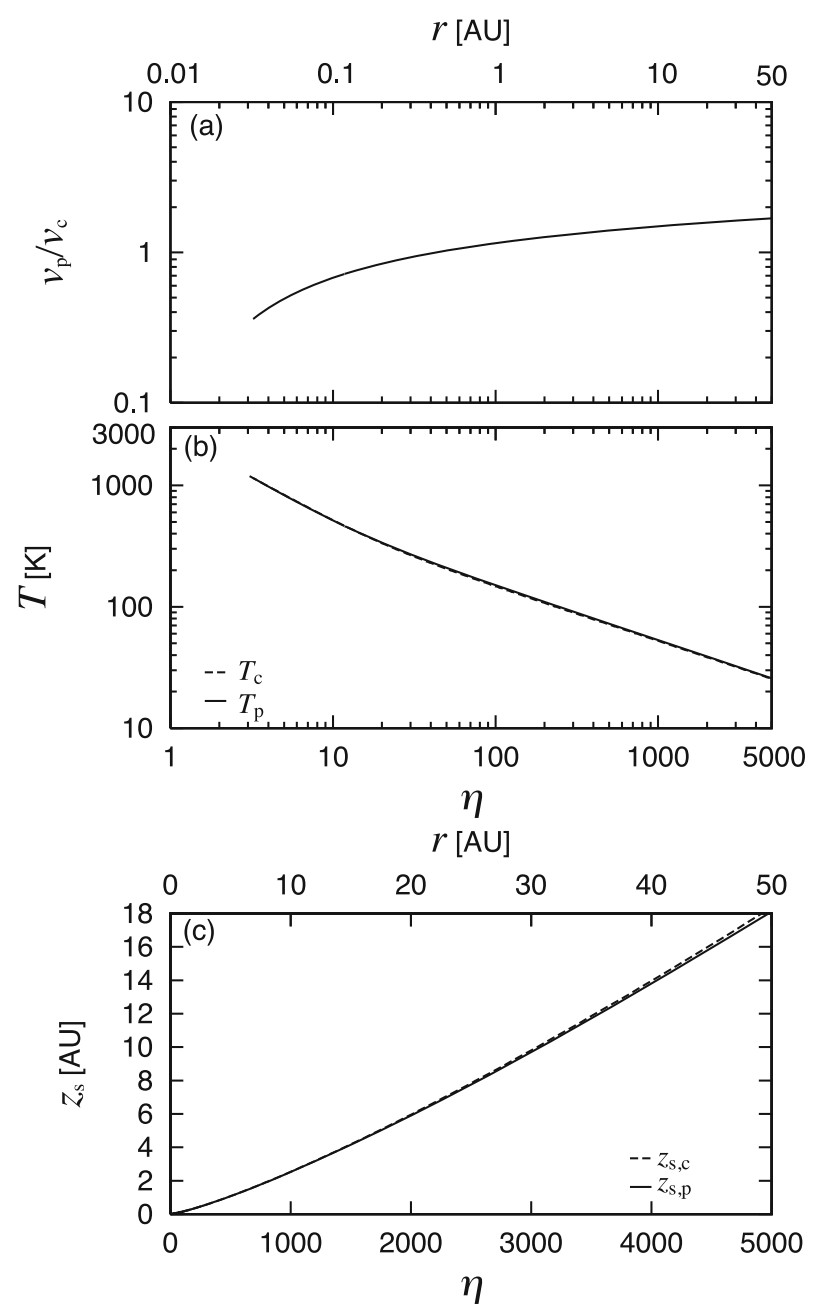

Fig. 6. The velocity, temperature and height based on Parker's solution. Panel (a) shows the velocity distribution, (b) the temperature distributions, and (c) the height of the disk for the case of $t_{*} / \beta=1 \times 10^{12} \mathrm{yr}$. Subscripts $\mathrm{p}$ and $\mathrm{c}$ denote the results calculated by Parker's solution and the constant velocity model in the case of $\beta(r)=1$, respectively.

trated in Figs. 7(a) and (b) for the case of $t_{*}=1 \times 10^{12} \mathrm{yr}^{-1}$, together with those for the constant velocity wind model with $\beta(r)=1$ (dashed curves). We can readily see from Fig. 7(a) that the two wind models give very similar results. The temperatures obtained from each of the two models coincide with each other almost completely. On the other hand, there is a difference in the height of the disk in the outer region beyond $r=10 \mathrm{AU}$ as presented in panel (b). This difference arises from the fact that the wind pressure, as mentioned earlier, is reduced in the case of $v_{\mathrm{w}} \propto r^{-1 / 2}$ compared with the case of constant $v_{\mathrm{w}}$.

We can explain the reason why the temperature does not change even if we use a different model for the wind velocity. The two basic equations described in Section 2 are subjected to slight modifications when the wind velocity is given by:

$$
v_{\mathrm{w}}=v_{\lambda}\left(\frac{R_{*}}{r}\right)^{\lambda},
$$

where $v_{\lambda}$ is the velocity at $r=R_{*}$. In place of Eqs. (12) and (15) we have, respectively:

$$
f^{2}=M \eta^{\gamma+\frac{1}{4}+\lambda} \sqrt{u} \exp (-s)
$$



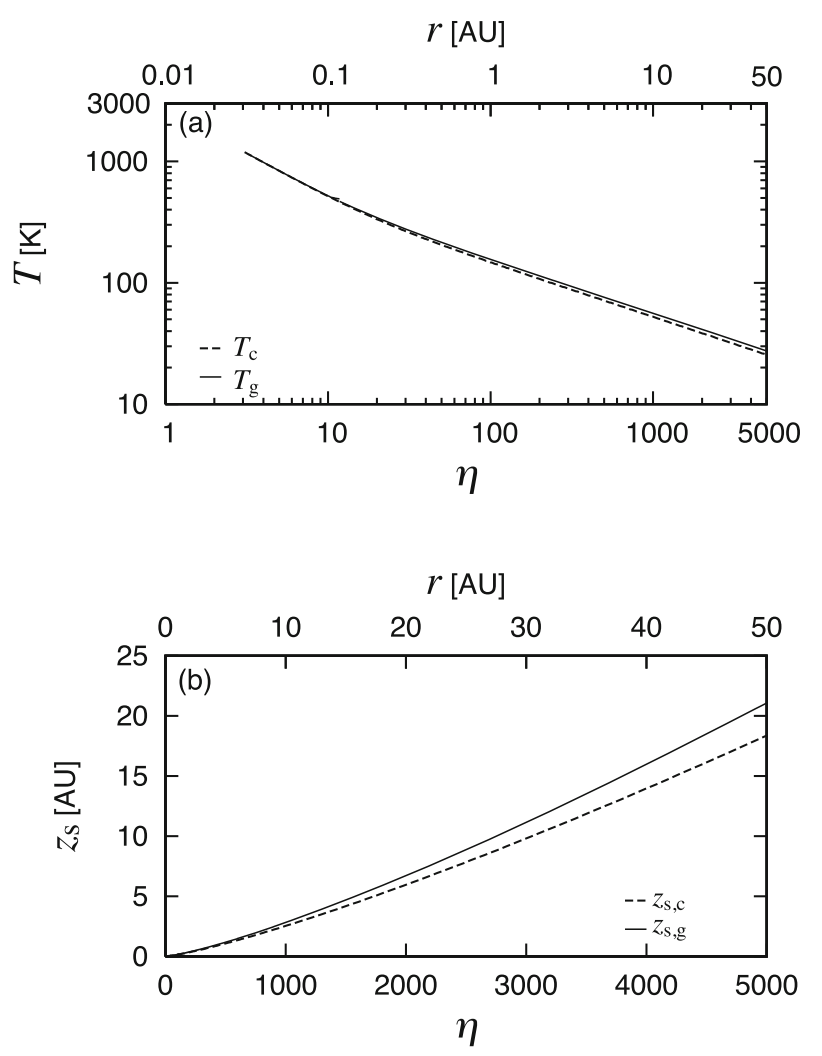

Fig. 7. The height and temperature of the disk for the case of $t_{*} / \beta=1 \times 10^{12} \mathrm{yr}$. Solid and dashed curves denote the results calculated by $v_{\mathrm{w}} \propto r^{-1 / 2}$ and the constant velocity model, respectively.

and

$$
M=2^{\frac{7}{8}} \sqrt{\pi} G \Sigma_{0} \frac{t_{*}}{v_{\lambda}}\left(\frac{T_{*}}{T_{\mathrm{g}}}\right)^{2}\left(\frac{R_{*}}{1 \mathrm{AU}}\right)^{\gamma+\lambda} .
$$

Thus, when the wind velocity is expressed as a power-law function of $r$, the disk model can be obtained simply from that of the constant velocity wind model by replacing $\gamma$ and $t_{*}$ with $\gamma+\lambda$ and $t_{*} v_{\mathrm{c}} / v_{\lambda}$, respectively $\left(v_{\mathrm{c}}\right.$ is the wind velocity given by Eq. (7) with $\beta=1$ ). Hence, as a result, we obtain almost the same solution for the wind models.

In any case, we can surely say that the difference in the wind velocity law does not appreciably affect the structure of the disk.

\section{Conclusions and Discussion}

In the present study, we calculate numerically the structure of a protoplanetary disk exposed to stellar wind, as well as the radiation from a central star changing the strength of the wind, the velocity law of the wind (i.e., the radial dependence of the wind velocity), and the mass distribution of the disk. The results obtained by a number of numerical calculations can be summarized as the following three conclusions:

(1) The temperature and the height of the disk surface are very insensitive to the adopted parameters describing the wind model (i.e., the wind strength and the velocity law of the wind) and the mass distribution model of the disk. In particular, in the inner region $(r \leq 0.1 \mathrm{AU})$ they are determined almost uniquely.

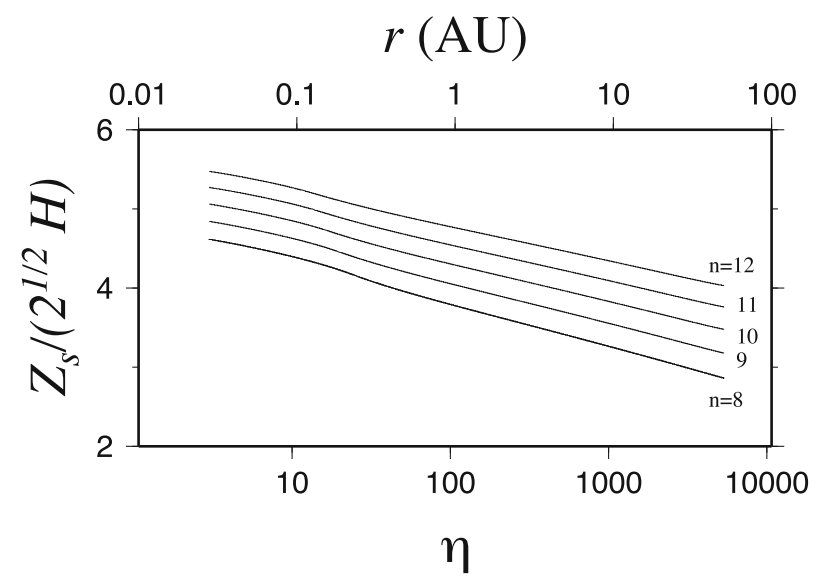

Fig. 8. The heights $z_{\mathrm{s}}$ normalized by the scale height, $\sqrt{2} H$. In the figure, each value of $\mathrm{n}$ has the same meaning as in Fig. 2.

(2) The flaring index of the disk, $f$, is suppressed by the dynamical pressure of the stellar wind and, as a result, is limited to a low level smaller than 0.1 . Furthermore, $f$ does not depend very much in general on the adopted wind model or on the mass distribution model of the disk. For example, even if the wind strength changes over four orders of magnitude, $f$ remains in a narrow variance of factor 2 .

(3) As long as we are concerned with the wind coexisting with the passive disk, both the temperature and the height of the disk depend only very weakly on the adopted parameters and are written approximately in the form of a power-law function of $r$. We obtain the temperature and height of the disk given by Eq. (25) and (26), respectively. To our regret, they are not expressed by a simple power-law function in the region $0.1 \mathrm{AU}<r<1 \mathrm{AU}$.

It is worthwhile making a comparison between our disk model and those of the previous works by K70 and CG97, in which they did not consider the effect of the stellar wind at all. For a precise comparison, the quantities specifying the central star are set to be equal to those of the present study; that is, $M_{*}=1 M_{\odot}, R_{*}=2 R_{\odot}$, and $T_{*}=4000$ $\mathrm{K}$. As mentioned before, the height, $z_{\mathrm{S}}$, is approximated well by a power-law function of the radial distance. The expressions predicted by our present model and the other models are given by:

$$
z_{\mathrm{s}}=\left\{\begin{array}{l}
0.15(r / 1 \mathrm{AU})^{1.2} \mathrm{AU} \text { for ours } r \geq 1 \mathrm{AU} \\
0.09(r / 1 \mathrm{AU})^{9 / 7} \mathrm{AU} \text { for } \mathrm{CG} 97 \\
0.02(r / 1 \mathrm{AU})^{9 / 7} \mathrm{AU} \text { for } \mathrm{K} 70 .
\end{array}\right.
$$

The difference in Eq. (32) becomes clearer by comparing $z_{\mathrm{s}} / \sqrt{2} H$ among the models where $\sqrt{2} H$ is the pressure scale height given by Eq. (5). As seen from Fig. 8, the ratio between $z_{\mathrm{s}}$ and $\sqrt{2} H$ changes from 2 to 5.5 in the disk. Contrary to K70, in which they assumed a priori that the height $z_{\mathrm{s}}$ is always equal to $\sqrt{2} H$, CG97 also showed that the ratio between the surface height and pressure scale height is almost equal to 4 .

The temperature of the disk predicted by the three models, including ours, are shown in Fig. 9. We can readily 


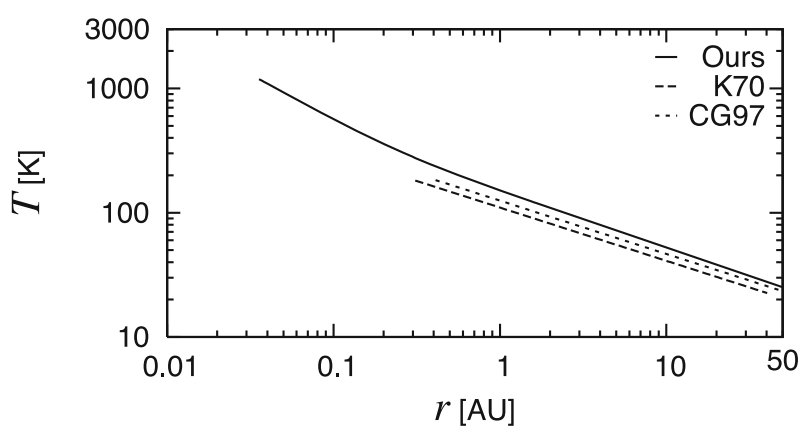

Fig. 9. Disk temperatures predicted by various models. Solid line denotes our result for the case of $t_{*} / \beta=1 \times 10^{10} \mathrm{yr}$. Dashed line and dotted line are temperature distributions of models of K70 and CG97, respectively. To compare the models under the same conditions, the temperature of K70 and that of CG97 are recalculated by using the parameters of the central star, $M_{*}=1 M_{\odot}, R_{*}=2 R_{\odot}$, and $T_{*}=4000 \mathrm{~K}$, after ours.

see from this figure that all the results are almost similar. For example, the temperatures at $r=1 \mathrm{AU}$ are 110 $\mathrm{K}, 125 \mathrm{~K}$, and $135 \mathrm{~K}$, respectively, for K70, CG97, and ours. The discrepancy in the temperatures comes mainly from the different choice of the height of the disk surface at which the disk receives the radiation from the central star. In our study, the height is determined by a dynamical balance and, as a result, the surface of the disk is the tallest of the three. Roughly speaking, the temperature of the disk becomes high when the height of the disk is great. Though the difference in the temperature among the three models is not so large, the results have an important significance in the sense that the radial position of the snow line is determined almost uniquely even if some uncertainties remain regarding the physical process governing the disk temperature.

Finally, we will add a few other remarks. As already pointed out in $\mathrm{Y} 07$, in addition to the direct radiative flux from the central star, the disk would receive radiative energy emitted from the surrounding diffuse gas, as well as heating energy due to cosmic rays. In the outer region $(r \geq 30$ $\mathrm{AU}$ ) of the disk, where the temperature is very low, such an additional energy flux cannot be ignored. Of course, the degree of the temperature increment in the outer region depends entirely on the physical configuration around the protoplanetary disk. In this sense, the temperature of the disk obtained in this study should be regarded as a minimum temperature in the outer region.

The strength of the stellar wind is assumed to be a constant temporarily in the present study. As mentioned in the first section, however, the stellar wind must change its strength with time. In Section 3, we found that the height of the disk changes very slightly with the adopted wind parameter $t_{*}$, as in the case of the temperature not being affected appreciably by $t_{*}$. Regarding the height of the surface, we can propose another kind of mechanism that is potentially important. Besides the shear region between the stellar wind and the disk, it is possible to generate a kind of instability, such as the Kelvin-Helmholtz instability. The wave produced on the contact surface will change the height of the surface and, as a result, change the heating efficiencies of the stellar radiation on the disk, too. It will be important to study the stability of the disk when the stellar wind changes its strength with time. This is a matter for further studies.

Acknowledgments. Authors have appreciated fruitful discussions with members in the Department of Earth and Planetary Sciences, Tokyo Institute of Technology. An advise given by Reviewer A helped authors to reconsider and reconfirm their conclusions. Authors have greatly appreciated to him or her, too.

\section{Appendix A.}

We show here the derivation of Eq. (10). First, we consider the radiation energy $d E^{\prime}$ emitted from an infinitesimal surface area of the central star $d S^{\prime}$ within the pencil designated by the solid angle $d \Omega$ and within the time interval $d t$. We can readily write down $d E^{\prime}$ as:

$$
\begin{aligned}
d E^{\prime} & =c \int_{0}^{\infty} \frac{8 \pi h v^{3}}{c^{3}} \frac{1}{\exp \left(h v / k T_{*}\right)-1} \boldsymbol{\Omega} \cdot d \boldsymbol{S}^{\prime} \frac{d \Omega}{4 \pi} d \nu d t \\
& =\frac{\sigma T_{*}^{4}}{\pi} \boldsymbol{\Omega} \cdot d \boldsymbol{S}^{\prime} d \Omega d t
\end{aligned}
$$

where $c, h, k$, and $\sigma$ are the light velocity, the Planck constant, the Boltzmann constant, and the Stefan-Boltzmann constant, respectively, and $T_{*}$ is the surface temperature of the central star. Furthermore, $\boldsymbol{\Omega}$ is the unit vector of the direction of the ray pencil given by:

$$
\Omega=\frac{r-x}{|r-x|} .
$$

The solid angle $d \Omega$ can be expressed in terms of the surface vector with a small area $d \boldsymbol{S}$ of the disk surface as (see Fig. A.1):

$$
d \Omega=-\frac{\boldsymbol{\Omega} \cdot d \boldsymbol{S}}{|\boldsymbol{r}-\boldsymbol{x}|^{2}} .
$$

Next, we evaluate the total radiation energy emitted from the possible surface of the central star and received on the disk surface $d \boldsymbol{S}$. In preparation of integrating over the surface of the central star, we express the vectors which appear in the above by their components. Namely, with the help of the geometry of the ray path shown in Fig. A.1, we find

$$
\left\{\begin{array}{l}
\boldsymbol{r}=r \boldsymbol{n}_{r}=r(0, \sin \phi, \cos \phi) \\
\boldsymbol{x}=R_{*} \boldsymbol{n}_{x}=R_{*}(\sin \theta \cos \varphi, \sin \theta \sin \varphi, \cos \theta) \\
d \boldsymbol{S}^{\prime}=d S^{\prime} \boldsymbol{n}_{x}=d S(\sin \theta \cos \varphi, \sin \theta \sin \varphi, \cos \theta) \\
d \boldsymbol{S}=d S \boldsymbol{n}_{S}=d S(0, \cos \Psi,-\sin \Psi)
\end{array}\right\},
$$

where $R_{*}$ is the radius of the central star and $\boldsymbol{n}_{i}$ is the unit vector of direction $i$. Using the above, we can readily obtain the following relations:

$$
\begin{gathered}
(\boldsymbol{r}-\boldsymbol{x}) \cdot d \boldsymbol{S}^{\prime}=r\left(\xi-\frac{R_{*}}{r}\right) d S^{\prime}, \\
(\boldsymbol{r}-\boldsymbol{x}) \cdot d \boldsymbol{S}=r \\
{\left[\sin (\phi-\Psi)+\frac{R_{*}}{r}(\sin \Psi \cos \theta\right.} \\
-\cos \Psi \sin \theta \sin \varphi)] d S,
\end{gathered}
$$

and

$$
(\boldsymbol{r}-\boldsymbol{x})^{2}=r^{2}\left(1-2 \xi \frac{R_{*}}{r}+\frac{R_{*}^{2}}{r^{2}}\right),
$$




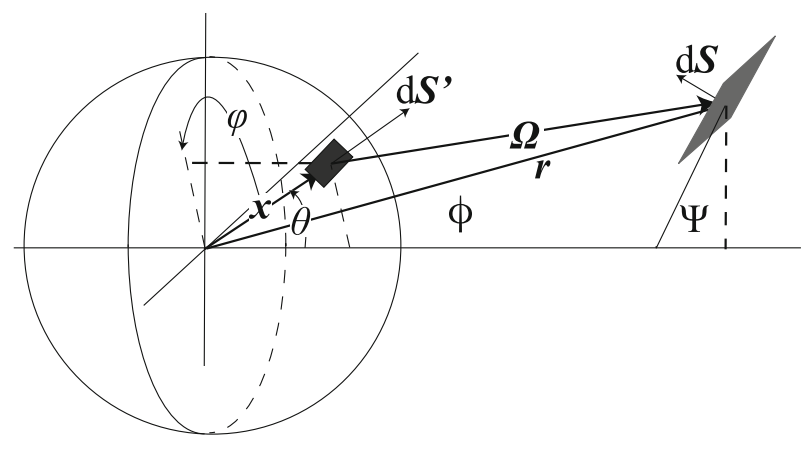

Fig. A.1. Geometry of radiation path from the blackbody.

where

$$
\xi=\cos \phi \cos \theta+\sin \phi \sin \theta \sin \varphi .
$$

Note that $d S^{\prime}$ in Eq. (A.5) can be expressed as:

$$
d S^{\prime}=R_{*}^{2} \sin \theta d \theta d \varphi .
$$

Here, we assume that the disk surface is exposed by the radiation emitted from the upper side of the central star; that is, the surface region upper from the equatorial plane of the disk. So, in integrating over the surface of the central star, we restrict ourselves to integration within the angles

$$
0<\theta<\frac{\pi}{2} \text { and } 0<\varphi<\pi
$$

The radiation energy $d E$ received by the disk surface $d S$ from the entire surface of the central star is given by:

$$
\begin{aligned}
d E & =\int_{\varphi} \int_{\theta} d E^{\prime}=\frac{\sigma T_{*}^{4}}{\pi} d t \int_{\varphi} \int_{\theta} \boldsymbol{\Omega} \cdot d \boldsymbol{S}^{\prime} d \Omega \\
& =-\frac{\sigma T_{*}^{4}}{\pi} d t \int_{0}^{\pi} \int_{0}^{\pi / 2} \frac{(\boldsymbol{r}-\boldsymbol{x}) \cdot d \boldsymbol{S}(\boldsymbol{r}-\boldsymbol{x}) \cdot d \boldsymbol{S}^{\prime}}{|\boldsymbol{r}-\boldsymbol{x}|^{4}} .
\end{aligned}
$$

Since $R_{*}<r,|\boldsymbol{r}-\boldsymbol{x}|^{-4}$ can be expanded as:

$$
\frac{1}{|\boldsymbol{r}-\boldsymbol{x}|^{4}}=\frac{1}{r^{4}}\left(1-2 \xi \frac{R_{*}}{r}+\frac{R_{*}^{2}}{r^{2}}\right)^{-2}=\frac{1}{r^{4}} \sum_{n=0}^{\infty} C_{n}^{2}(\xi)\left(\frac{R_{*}}{r}\right)^{n},
$$

where $C_{n}^{m}(\xi)$ is the Gegenbauer polynomial. By the use of Eqs. (A.5), (A.6), and (A.11), we can write down the integrand in Eq. (A.11) in the following form:

$$
\begin{aligned}
& \frac{(\boldsymbol{r}-\boldsymbol{x}) \cdot d \boldsymbol{S}(\boldsymbol{r}-\boldsymbol{x}) \cdot d \boldsymbol{S}^{\prime}}{|\boldsymbol{r}-\boldsymbol{x}|^{4}} \\
& =\left(\frac{R_{*}}{r}\right)^{2} \sum_{n=0}^{\infty} C_{n}^{2}(\xi) \sin (\phi-\Psi)\left(\frac{R_{*}}{r}\right)^{n} \frac{d S^{\prime}}{R_{*}^{2}} d S \\
& +\left(\frac{R_{*}}{r}\right)^{2} \sum_{n=0}^{\infty}[(\sin \Psi \cos \theta-\cos \Psi \sin \theta \sin \varphi) \xi \\
& -\sin (\phi-\Psi)]\left(\frac{R_{*}}{r}\right)^{n+1} \frac{d S^{\prime}}{R_{*}^{2}} d S \\
& -\left(\frac{R_{*}}{r}\right)^{2} \sum_{n=0}^{\infty}(\sin \Psi \cos \theta-\cos \Psi \sin \theta \sin \varphi) \\
& \times\left(\frac{R_{*}}{r}\right)^{n+2} \frac{d S^{\prime}}{R_{*}^{2}} d S .
\end{aligned}
$$

In the present study, we assume that $R_{*} \ll r$ and, thus, we retain only second- and third-order terms of $R_{*} / r$ in the above equation, namely:

$$
\begin{aligned}
& \frac{(\boldsymbol{r}-\boldsymbol{x}) \cdot d \boldsymbol{S}(\boldsymbol{r}-\boldsymbol{x}) \cdot d \boldsymbol{S}^{\prime}}{|\boldsymbol{r}-\boldsymbol{x}|^{4}} \\
& =C_{0}^{2}(\xi) \sin (\phi-\Psi)\left(\frac{R_{*}}{r}\right)^{2} \frac{d S^{\prime}}{R_{*}^{2}} d S \\
& +\left\{\left(C_{1}^{2}(\xi) \xi-C_{0}^{2}(\xi)\right) \sin (\phi-\Psi)\right. \\
& \left.+C_{0}^{2}(\xi)(\sin \Psi \cos \theta-\cos \Psi \sin \theta \sin \varphi) \xi\right\}\left(\frac{R_{*}}{r}\right)^{3} \frac{d S^{\prime}}{R_{*}^{2}} d S .
\end{aligned}
$$

Noting the relations:

$$
\begin{aligned}
C_{2}^{1}(\xi)= & \xi C_{1}^{2}(\xi)-C_{0}^{2}(\xi), \quad C_{0}^{m}(\xi)=1, \\
& \text { and } \quad C_{2}^{1}(\xi)=4 \xi^{2}-1,
\end{aligned}
$$

we obtain

$$
\begin{aligned}
& \frac{(\boldsymbol{r}-\boldsymbol{x}) \cdot d \boldsymbol{S}(\boldsymbol{r}-\boldsymbol{x}) \cdot d \boldsymbol{S}^{\prime}}{|\boldsymbol{r}-\boldsymbol{x}|^{4}} \\
& =\xi \sin (\phi-\Psi)\left(\frac{R_{*}}{r}\right)^{2} \frac{d S^{\prime}}{R_{*}^{2}} d S+\left\{\left(4 \xi^{2}-1\right) \sin (\phi-\Psi)\right. \\
& +(\sin \Psi \cos \theta-\cos \Psi \sin \theta \sin \varphi) \xi\}\left(\frac{R_{*}}{r}\right)^{3} \frac{d S^{\prime}}{R_{*}^{2}} d S .
\end{aligned}
$$

Integrating Eq. (A.16) over $\theta$ and $\varphi$, we readily find

$$
\begin{aligned}
& \int_{0}^{\pi} \int_{0}^{\pi / 2} \frac{(\boldsymbol{r}-\boldsymbol{x}) \cdot d \boldsymbol{S}(\boldsymbol{r}-\boldsymbol{x}) \cdot d \boldsymbol{S}^{\prime}}{|\boldsymbol{r}-\boldsymbol{x}|^{4}} \\
& =-\frac{\pi}{2} \sin (\Psi-\phi)(\cos \phi+\sin \phi)\left(\frac{R_{*}}{r}\right)^{2} d S \\
& -\left[\frac{8}{3} \sin 2 \phi \sin (\Psi-\phi)+\frac{2}{3} \cos (\Psi+\phi)\right]\left(\frac{R_{*}}{r}\right)^{3} d S .
\end{aligned}
$$

The radiation energy $d E$ is absorbed on the disk surface with the normal vector $d \boldsymbol{S}$ and is reemitted as blackbody radiation during the time interval $d t$. Thus, we have for the balance equation of the radiation energy on the disk surface

$$
d E=\sigma T^{4} d S d t
$$

where $T$ is the temperature of the disk surface. From Eqs. (A.11), (A.17), and (A.18), we arrive at the following equation which gives the temperature of the disk surface:

$$
\begin{aligned}
\sigma T^{4}= & \frac{\sigma T_{*}^{4}}{\pi}\left(\frac{R_{*}}{r}\right)^{2}\left\{\frac{\pi}{2} \sin (\Psi-\phi)(\cos \phi+\sin \phi)\right. \\
& \left.+\frac{R_{*}}{r}\left[\frac{8}{3} \sin 2 \phi \sin (\Psi-\phi)+\frac{2}{3} \cos (\Psi+\phi)\right]\right\} .
\end{aligned}
$$

Now, we consider the geometry of the contact surface between the disk and the wind. For the cylindrical coordinates 
$(x, z)$ of which the origin is at the center of the central star (see figure 1 in Y07), two angles, $\phi$ and $\Psi$, are defined by:

$$
\phi=\tan ^{-1}\left(\frac{z_{\mathrm{s}}}{r}\right) \quad \text { and } \quad \Psi=\tan ^{-1}\left(\frac{d z_{\mathrm{s}}}{d r}\right),
$$

where $z_{\mathrm{s}}$ is the height of the disk. Using the two angles, the flaring index, $f$, is given by:

$$
f=\sin (\Psi-\phi) .
$$

Furthermore, the following equations can be derived:

$$
\sin \phi=\frac{z_{\mathrm{s}} / r}{\sqrt{1+\left(z_{\mathrm{s}} / r\right)^{2}}}, \cos \phi=\frac{1}{\sqrt{1+\left(z_{\mathrm{s}} / r\right)^{2}}},
$$

$\sin \Psi=\frac{d z_{\mathrm{s}} / d r}{\sqrt{1+\left(d z_{\mathrm{s}} / d r\right)^{2}}}$, and $\cos \Psi=\frac{1}{\sqrt{1+\left(d z_{\mathrm{s}} / d r\right)^{2}}}$.

We rewrite Eq. (A.19) by using Eqs. (A.21) and (A.22) as:

$$
\begin{aligned}
\sigma T^{4}= & \frac{\sigma T_{*}^{4}}{\pi} \frac{R_{*}^{2}}{r^{2}}\left\{\frac{\pi}{2} f \frac{1+z_{\mathrm{s}} / r}{\sqrt{1+\left(z_{\mathrm{s}} / r\right)^{2}}}\right. \\
& +\frac{2 R_{*}}{3 r}\left[8 f \frac{z_{\mathrm{s}} / r}{1+\left(z_{\mathrm{s}} / r\right)^{2}}\right. \\
& \left.\left.+\frac{1-\left(z_{\mathrm{s}} / r\right)\left(d z_{\mathrm{s}} / d r\right)}{\sqrt{1+\left(d z_{\mathrm{s}} / d r\right)^{2}} \sqrt{1+\left(z_{\mathrm{s}} / r\right)^{2}}}\right]\right\} .
\end{aligned}
$$

Finally, we obtain the radiative equilibrium condition as Eq. (10).

\section{References}

Beckwith, S. V. W., A. I. Sargent, R. S. Chini, and R. Güsten, A survey for circumstellar disks around young stellar objects, Astron. J., 99, 924945, 1990.

Cabrit, S., S. Edwards, S. E. Strom, and K. M. Strom, Forbidden-line emission and infrared excess in T Tauri stars: Evidence for accretiondrive mass loss?, Astorophys. J., 354, 687-700, 1990.

Calvet, N., Properties of the wind of T Tauri stars, IAUS, 182, 417-432, 1997.

Calvet, N., L. Hartmann, and R. Hewett, Winds from T Tauri stars. II. Balmer line profiles for inner disk winds, Astrophys. J., 386, 229-238, 1992.

Carr, J. S., Near-infrared CO emission in young stellar objects, Astrophys $J ., \mathbf{3 4 5}, 522-535,1989$.

Chiang, E. I. and P. Goldreich, Spectral energy distributions of T Tauri stars with passive circumstellar disks, Astrophys. J., 490, 368-376, 1997.

Chiang, E. I., M. K. Joung, M. J. Creech-Eakman, C. Qi, J. E. Kessler, G. A. Blake, and E. F. van Dishoeck, Spectral energy distributions of passive T Tauri and Herbig Ae disks: grain mineralogy, parameter dependences, and comparison with infrared space observatory LWS observations, Astrophys. J., 547, 1077-1089, 2001.

D’Alessio, P., J. Canto, L. Hartmann, N. Calvet, and S. Lizano, On the thermal stability of irradiation-dominated pre-main-sequence disks, Astrophys. J., 511, 896-903, 1999.

Dullemond, C. P., Are passive protostellar disks stable to self-shadowing?, Astron. Astrophys., 361, L17-L20, 2000.

Dullemond, C. P. and C. Domink, Flaring vs. self-shadowed disks: The SEDs of Herbig Ae/Be stars, Astron. Astrophys., 417, 159-168, 2004.

Dullemond, C. P., C. Domink, and A. Natta, Passive irradiation circumstellar disks with an inner hole, Astrophys. J., 560, 957-969, 2001.
Edwards, S., P. Hartigan, L. Ghandour, and C. Andrulis, Spectroscopic evidence for magnetospheric accretion in classical T Tauri stars, Astron. $J .$, 108, 1056-1070, 1994.

Garaud, P. and D. N. C. Lin, The effect of internal dissipation and surface irradiation on the structure of disks and the location of the snow line around sun-like stars, Astrophys. J., 654, 606-624, 2007.

Greene, T. P. and M. Meyer, An infrared spectroscopic survey of the $\rho$ Ophiuchi young stellar cluster: Masses and ages from the H-R diagram, Astrophys. J., 450, 233-244, 1995.

Hartigan, P., S. Edwards, and L. Ghandour, Disk Accretion and loss from young stars, Astrophys. J., 452, 736-768, 1995.

Hartmann, L. and S. J. Kenyon, Optical veiling, disk accretion, and the evolution of T Tauri stars, Astrophys. J., 349, 190-196, 1990.

Hartmann, L., N. Calvet, E. Avrett, and R. Loeser, Winds from T Tauri stars. I. Spherically symmetric models, Astrophys. J., 349, 168-189, 1990.

Hayashi, C., Structure of the solar nebula, growth and decay of magnetic field, and effects of magnetic and turbulent viscosities on the nebula, Prog. Theor. Phys. Suppl., 70, 35-53, 1981.

Hayashi, C., K. Nakazawa, and Y. Nakagawa, Formation of the Solar System, in Protostars and Planets II, edited by Black, D. C. and M. S. Matthews, pp. 1100-1153, Univ. Arizona Press, Tucson, 1985.

Jonkheid, B., F. G. A. Faas, G.-J. van Zadelhoff, and E. F. van Dishoeck, The gas temperature in flaring disks around pre-main sequence stars, Astron. Astrophys., 428, 511-521, 2004.

Kamp, I. and C. P. Dullemond, The gas temperature in the surface layers of protoplanetary disks, Astrophys. J., 615, 991-999, 2004.

Kenyon, S. J. and L. Hartmann, Pre-main-sequence evolution in the Taurus-Auriga molecular cloud, Astrophys. J. Suppl., 101, 117-171, 1995.

Kuhi, L., Mass loss from T Tauri stars, Astrophys. J., 140, 1409-1432, 1964.

Kusaka, T., T. Nakano, and C. Hayashi, Growth of solid particles in the primordial solar nebula, Progr. Theor. Phys., 44, 1580-1595, 1970.

McCaughrean, M. J. and C. R. O’Dell, Direct imaging of circumstellar disks in the Orion nebula, Astron. J., 111, 1977-1987, 1996.

Miyake, K. and Y. Nakagawa, Dust particle settling in passive disks around T Tauri stars: Models and IRAS Observations, Astrophys. J.,441, 361384, 1995.

Parker, E. N., The hydrodynamic theory of solar corpuscular radiation and stellar winds, Astrophys. J., 132, 821-866, 1960.

Parker, E. N., Dynamical properties of stellar coronas and stellar winds.I. Integration of the momentum equation, Astrophys. J., 139, 72-92, 1964.

Sasselov, D. D. and M. Lecar, On the snow line in dusty protoplanetary disks, Astrophys. J., 528, 995-998, 2000.

Shu, F. H., F. C. Adams, and S. Lizano, Star formation in molecular clouds-Observation and theory, Ann. Rev. Astron. Astrophys., 25, 23 81, 1987.

Strom, K. M., S. E. Strom, S. Edwards, S. Cabrit, and M. F. Skruskie, Circumstellar material associated with solar-type pre-main-sequence stars: A possible on the timescale for planet building, Astron. J., 97, 1451$1470,1989$.

Watanabe, S. and D. N. C. Lin, Thermal wave in irradiated protoplanetary disks, Astron. J., 672, 1183-1195, 2008.

White, J. S. and L. A. Hillenbrand, On the evolutionary status of Class I stars and Herbig-Haro energy sources in Taurus-Auriga, Astorophys. J., 616, 998-1032, 2004.

Wood, B. E., H.-R. Muller, G. P. Zank, and J. L. Linsky, Measured massloss rates of solar-like stars as a function of age and activity, Astrophys. J., 574, 412-425, 2002.

Yun, Y. S., H. Emori, and K. Nakazawa, Thermal and hydrostatic structure of the protoplanetary nebula exposed to stellar radiation and stellar wind from the central star, Earth Planets Space, 59, 631-643, 2007.

Yun, Y. S., H. Emori, and K. Nakazawa, Thermal and hydrostatic structure of the protoplanetary nebula exposed to stellar radiation and stellar wind from the central star, ERRATA, Earth Planets Space, 62(no.6), 551$552,2010$.

Y. S. Yun, H. Emori (e-mail: emori@ts.shumei-u.ac.jp), and K. Nakazawa 